\title{
A Case Report of Stroke in a Young Recreational Drug Abuser with Left Ventricular Apical Myxoma with Lupus Nephritis, Secondary Antiphospholipid Syndrome and Homocysteinemia
}

Rumana Habib ${ }^{1}$, Reezwan Islam², Md.Babul Miah³ ${ }^{3}$ Md. Rashedul Islam ${ }^{4}$ Aminur Rahman ${ }^{5}$, Nirmalendu Bikash Bhowmik ${ }^{6}$

\begin{abstract}
:
In young adults $20 \%-47 \%$ cases of stroke is due to cardiac embolism. Cardiac Myxoma is an infrequent cause of embolic stroke in young. We are reporting a case of a ischemic stroke in a young adult of 34 years with a history of recreational drug use who was eventually diagnosed with Left Ventricular Apical Myxoma,
\end{abstract}

Systemic Lupus Erythematous (SLE), Lupus Nephritis, Secondary Anti phospholipid Syndrome, Hyper homocysteinemia, and Dyslipidemia.

Keywords: Stroke, Young Adult, Myxoma, Lupus Nephritis, Homocysteinemia

\section{Introduction:}

Stroke is often considered a disease of older people, but an estimated $10 \%$ of patients with stroke are younger than 50 years $^{1}$. Many of them have no risk factors for atherosclerosis and no ultimate clear etiological diagnosis even after a thorough investigation. This diagnostic challenge is one of the main scopes of studying and researching mechanisms of brain ischemia in young adults in addition to the dramatic personal, familial, and socio-economic consequences by affecting individuals at the top of their productive age.

1. Assistant Professor, Department of Neurology, BIRDEM General Hospital, Dhaka.

2. Resident Medical Officer, Department of Neurology, BIRDEM General Hospital, Dhaka.

3. Senior Medical Officer, Department of Cardiology, BIRDEM General Hospital, Dhaka.

4. Registrar, Department of Neurology, BIRDEM General Hospital, Dhaka.

5. Consultant, Department of Neurology, BIRDEM General Hospital, Dhaka.

6. Professor, Department of Neurology, BIRDEM General Hospital, Dhaka.

Address of Correspondence: Dr. Rumana Habib, Assistant Professor, Department of Neurology, BIRDEM General Hospital, Dhaka, E-mail:drhrumana4@gmail.com
Cardioembolism and cervicocephalic arterial dissection have been established as principal etiological factors of IS in young adults ${ }^{2}$, The proportion of cardioembolic strokes in young adults varies from $20 \%$ to one third ${ }^{3,4}$.

Considered an unusual cause of IS in the young two decades $\mathrm{ago}^{5}$, atherosclerosis has gaining projection by recent reports of significant raise in traditional risk factors as hypertension, diabetes, obesity, dyslipidemia and tabagism among hospitalized adolescents and young adults 6 .

Only $1-4 \%$ of IS are related to acquired and genetic thrombophilias, but these numbers seem higher in young adults $^{7}$. The most common acquired thrombophilia associated to IS in the young is antiphospholipid syndrome. Antiphospholipid antibodies, particularly lupus anticoagulant, are an independent risk factor for IS in young adults ${ }^{8}$. Genetic prothrombotic states play an important role in young patients with cerebral venous thrombosis, but thrombophilia alone rarely causes arterial occlusions ${ }^{9}$.

We herein report a case of a young adult male who was diagnosed as having Ischemic stroke with right sided hemiplegia and Left Ventricular Apical Myxoma, as well as multiple other comorbidities, each of which are capable of causing recurrent stroke. 


\section{Case report:}

A 34 year old non diabetic, normotensive gentleman presented on $17^{\text {th }}$, April 2016 with sudden onset right sided weakness and speech difficulty for twelve hours. He had no loss of vision, diplopia or dizziness. He gave no history of headache, vomiting, convulsion or loss of consciousness. On further query, he denied having any rash, arthralgia, chest pain, palpitation, breathlessness, or syncope. He had no relevant family history, but he was a chain smoker (20 pack year) and had a history of Cannabis abuse. Examination revealed he was drowsy with normal vital signs. Neurological examination showed nonfluent aphasia and right hemiplegia .Examination of the other systems revealed no abnormality.

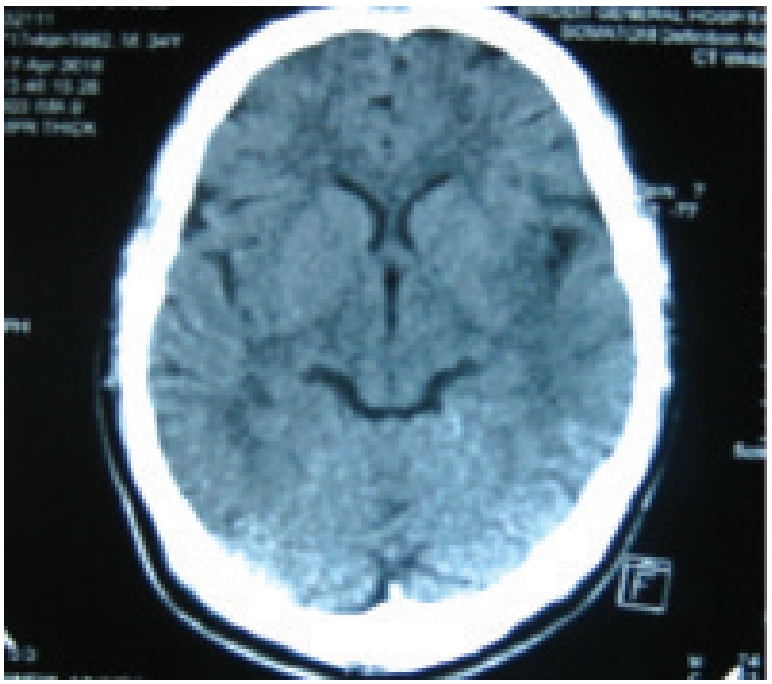

CT scan of the brain (Figure 1) showed early signs of infarction the loss of 'insular ribbon' sign. MRI of the brain (Figure 2) showed an embolic acute large left MCA territory infarct with midline shifting and effacement of the left lateral ventricles.

ECG (Figure 3) showed sinus rhythm with anterolateral ischaemia and Chest X-Ray was normal. CBC: $\mathrm{Hb}-11.5 \mathrm{~g} /$

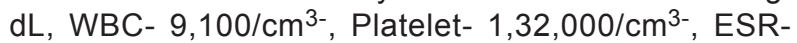
$42 \mathrm{~mm}$ in $1^{\text {st }}$ hour. Urine R/M/E revealed Pus cell:1-2/ HPF, Epithelial cell:0-3/HPF, RBC: Occasional, Protein: ++ . S. electrolytes, renal function tests and liver function tests were within normal range.

Evaluation of the patient by Transthoracic echocardiography (Figure 4) revealed an inhomogeneous, contractile,

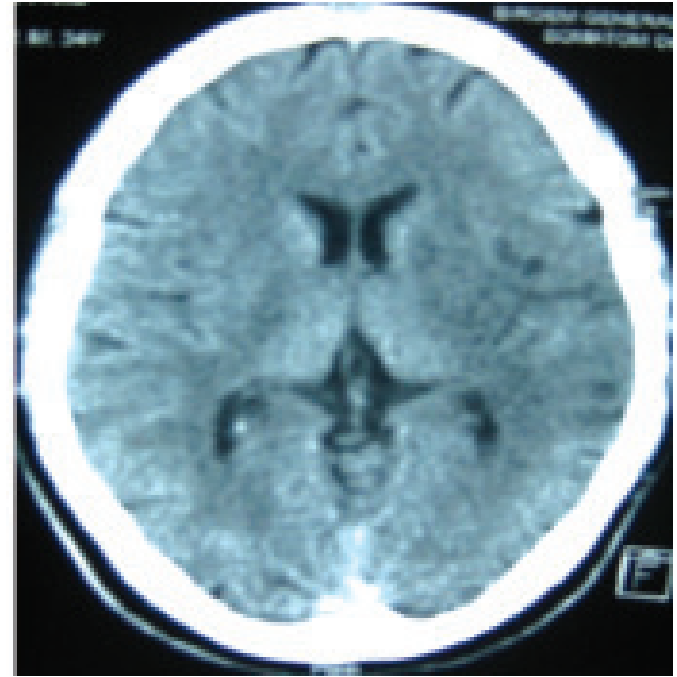

Fig.-1: CT Scan Brain showing the loss of 'insular ribbon' sign on left cerebral hemisphere
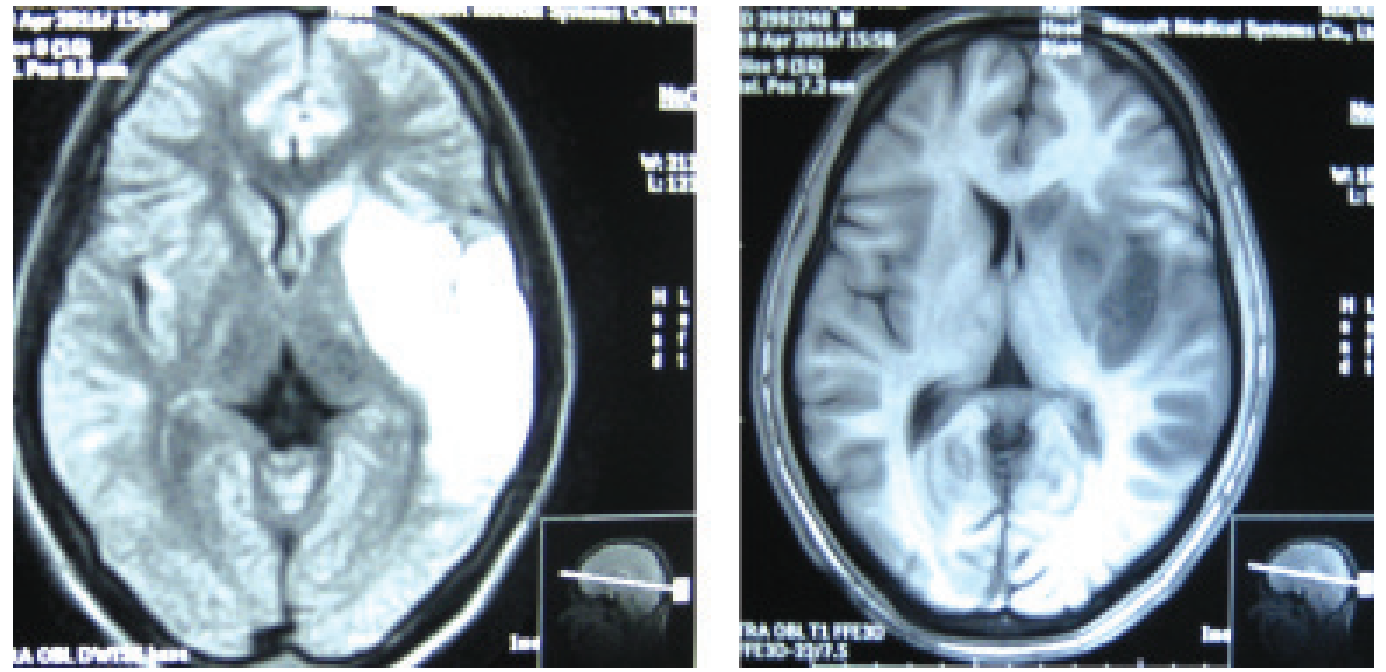

Fig.-2: MRI of Brain showing acute large left MCA territory infarction with midline shifting and effacement of the left lateral ventricles. 


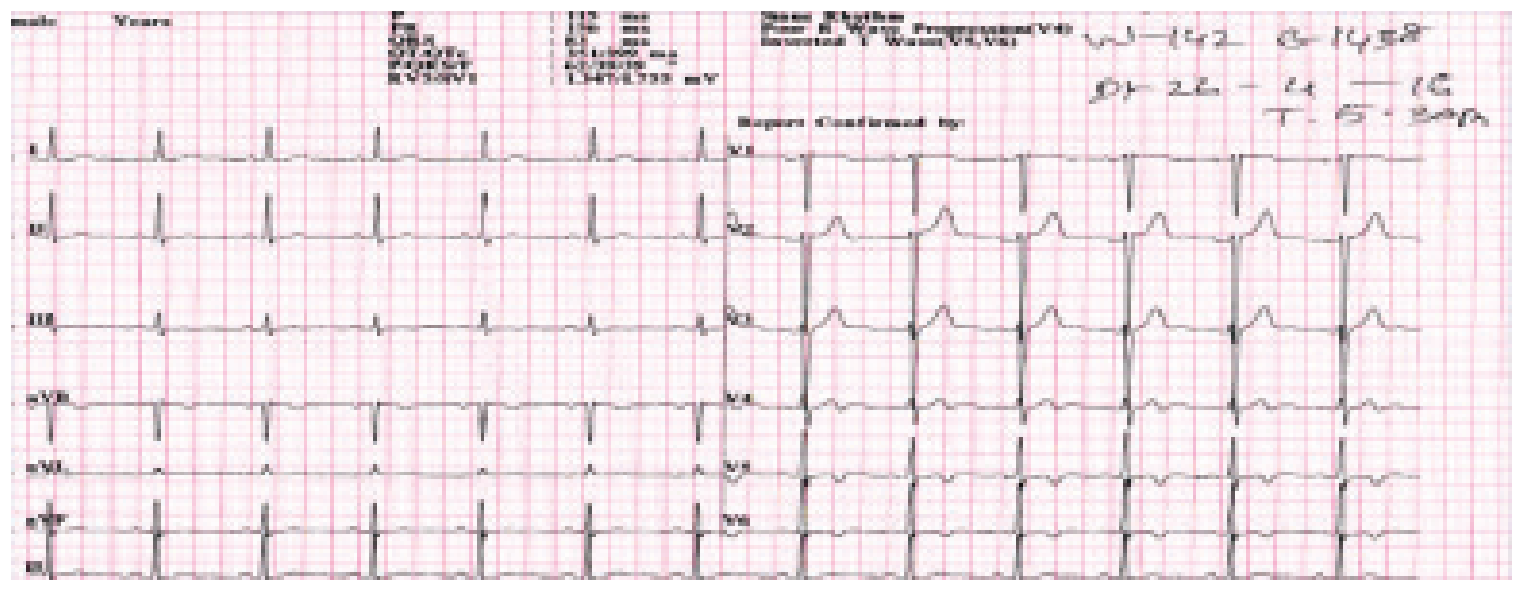

Fig.-3: ECG showing normal sinus rhythm and anterolateral ischaemia.
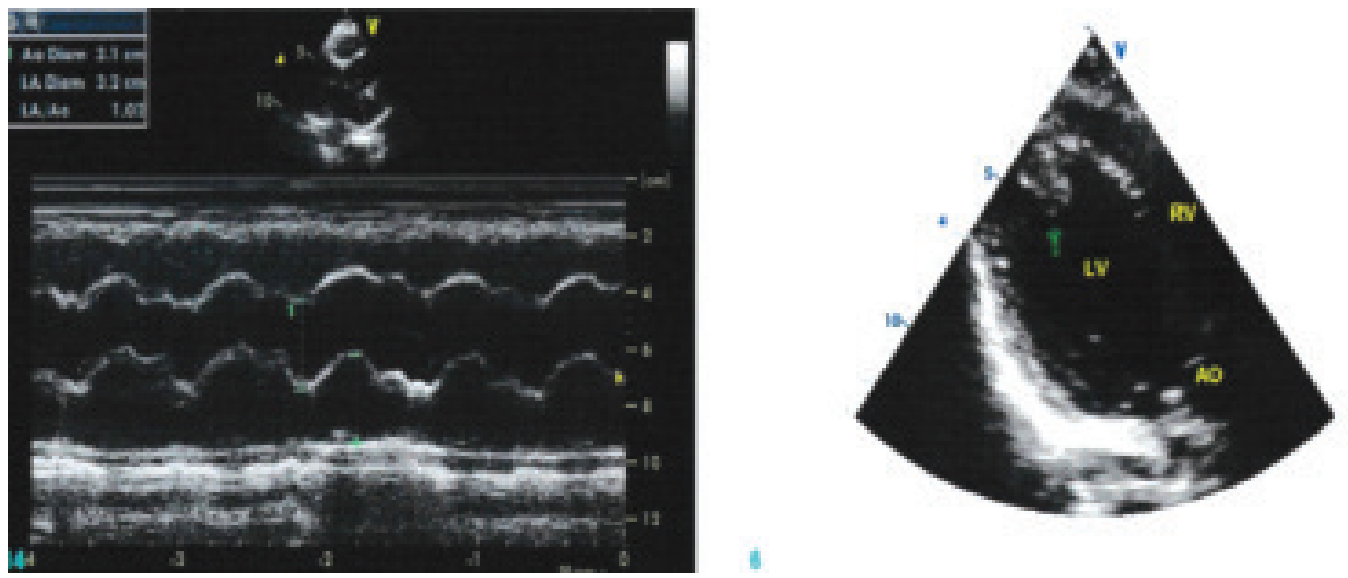

Fig.-4: Transthoracic echocardiography showing a pedunculated heterogeneous mass (arrow) in the left ventricle.

pedunculated, echogenic mass (measuring $19 \times 16 \mathrm{~mm}$ ) attached to the apical septum of left ventricle strongly suggestive of Left ventricular Apical Myxoma. No LV regional wall motion abnormality was detected and LV ejection fraction of $65 \%$.

Following a consultation with Cardiac Surgeon, a Transoesophageal echocardiography (TEE) was done which showed the Myxoma with non-organized thrombus or vegetation attached with it (Figure 5).

Investigations to detect risk factors for stroke in young adults done in our patient were S. Homocysteine: 30.1 $\mu \mathrm{mol} / \mathrm{l}, \mathrm{VDRL}$ : non-reactive, TPHA: non-reactive, Anti phospholipid antibody: $40 \mathrm{U} / \mathrm{ml}$ (Positive), ANA: Positive (>1.5), Anti ds DNA: Positive $27 \mathrm{U} / \mathrm{ml}$ (>25 U/ml). Toxicology screen for Urine was Positive for Cannabinoids. Further investigations included PBF which showed nonspecific morphology with neutrophilia and thrombocytopenia. Blood culture showed no growth.
PT: $13.4 \mathrm{sec}$; INR: 1.1 . Doppler study of neck vessels revealed: Bilateral mild atherosclerosis with no limitation of flow. The patient was not evaluated for Protien $\mathrm{C}$ and Proteins deficiency.

Macroscopic hematuria and high titre of ANA and positive ds DNA warrented further investigation. Phase contrast microscopy of urine was done and it showed 3-5 RBC/ HPF, (10\% dysmorphic). 24 hours urinary total protein estimation was $1.00 \mathrm{gm} /$ day, Ccr: $62 \mathrm{ml} / \mathrm{min}$. USG of whole abdomen showed mild swelling of both kidneys. Renal biopsy (Figure 6) revealed mesangial proliferative lupus nephritis (Class-II Lupus nephritis). Serum C3 and C4 levels were within normal range. Vasculitis markers including c-ANCA and p-ANCA were negative.

The patient was initially treated with I.V Mannitol, Aspirin, Pantoprazole, Losartan Potassium, Folic acid, Vitamin B6, Vitamin B12 combination, Atorvastatin and daily physiotherapy. The final treatment was modified after 

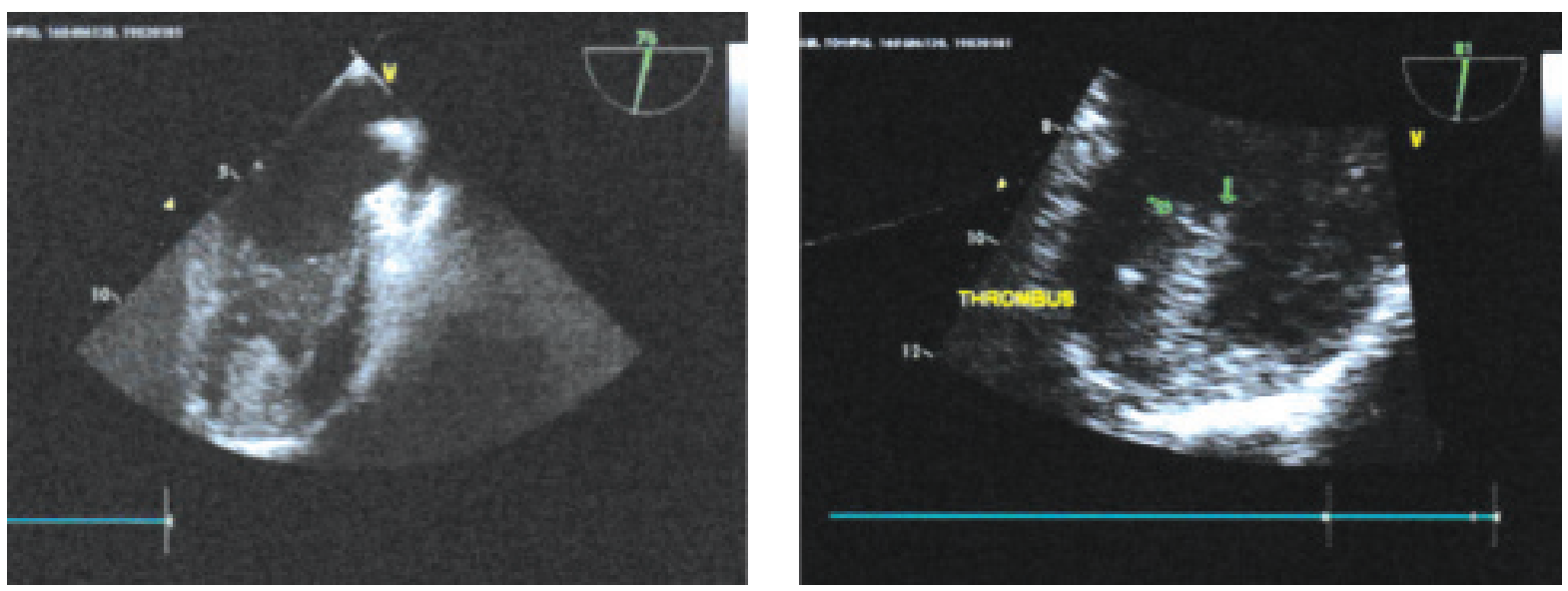

Fig.-5: Transeosophageal Echo showing Left ventricular apical Myxoma with non-organized thrombus or vegetation.
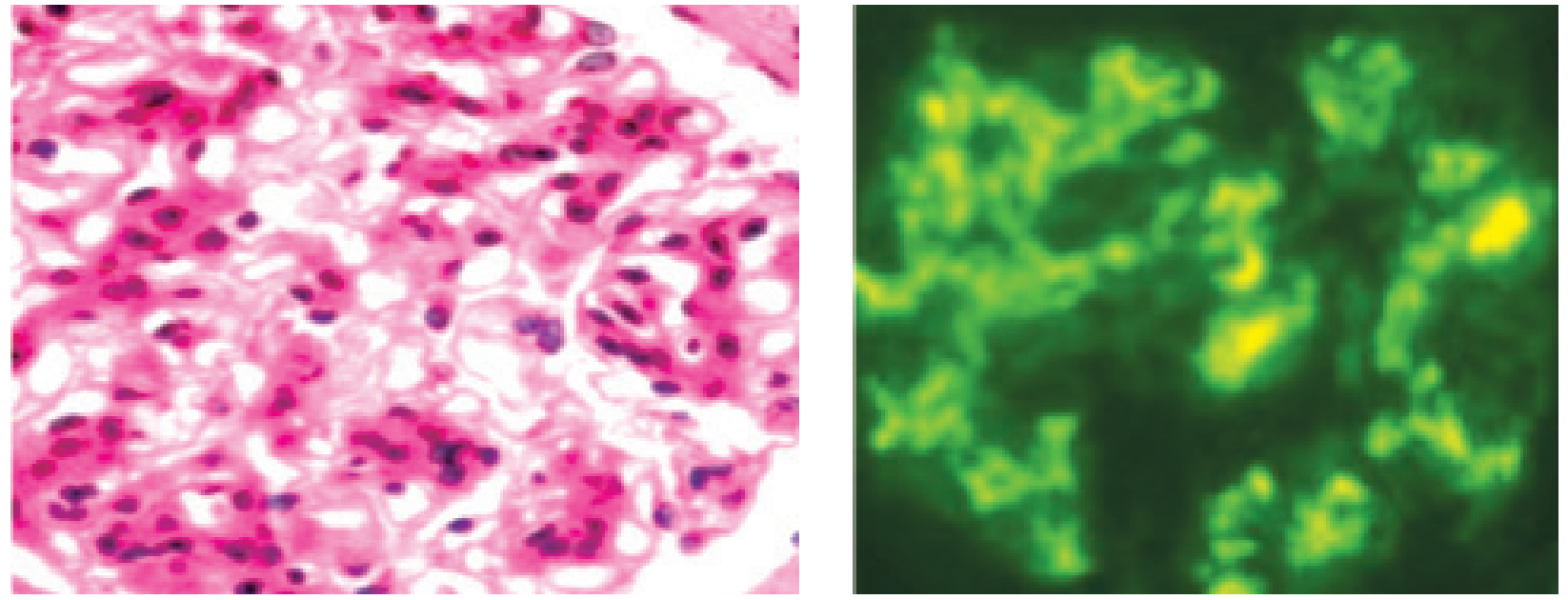

Fig.-6: Mesangial proliferative lupus nephritis (Class-II lupus nephritis).

Consultation with Cardiac Surgeon and Nephrologist and it included Warfarin keeping international normalized ratio value between 2 and 3 and Hydroxychloroquine Sulphate (200mg) once daily. After improvement of general condition he was discharged with advice to follow up in the OPD with PT and INR, creatinine and Urine R/M/E.

The patient was discharged home with advice to follow up with Echocardiography, PT and INR, S. creatinine and Urine $\mathrm{R} / \mathrm{M} / \mathrm{E}$.

Echocardiogram 6 weeks post discharge revealed a sessile LV apical myxoma of $2 \mathrm{~cm}^{2}$ in size (FIGURE 7). Cardiac surgery consultation was taken at that time and the Surgeon advised to follow-up the patient for another 6 months to see the progression of LV mass.

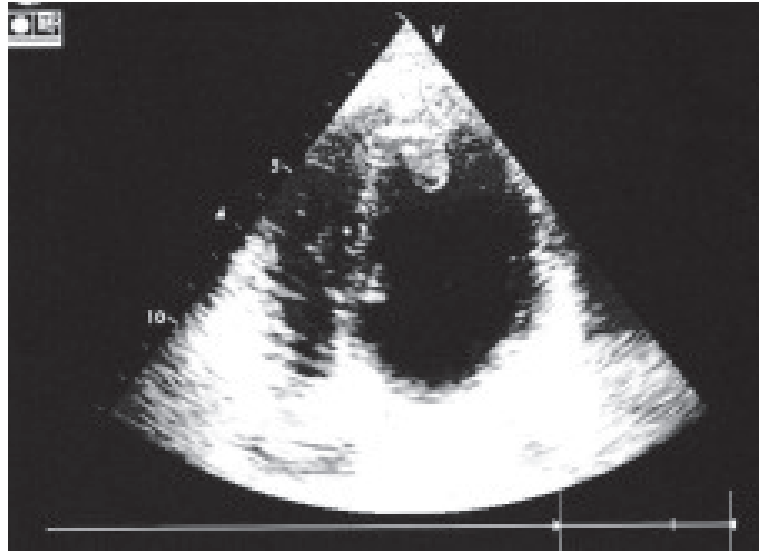

Fig.-7: Follow up echocardiogram 


\section{Discussion:}

Cardiac myxomas, although uncommon are one of commonest primary cardiac tumours and account for $\sim 50 \%$ primary benign cardiac tumours. ${ }^{10}$ The majority of myxomas are in the left atrium ( $75 \%)$ followed by the right atrium (20\%). LV myxomas account for only $2.5 \%$ of cases $^{11}$. The first description of a primary intracardiac tumor was in 1559 , located in the left ventricle ${ }^{12}$. In a review of literature, Mazer and Harrigan reported the first case of LV myxoma diagnosed by $2 \mathrm{D}$ echocardiography in $1982{ }^{13}$. Only 37 cases of left ventricular myxomas have been reported up to $1996{ }^{14}$ and 72 cases up to $2014^{15}$. In this respect, our case is an extremely rare one.

Myxomas develop in all age groups. Myxomas are found particularly frequently between the $3 r d$ and $6^{\text {th }}$ decades of life ${ }^{16}$.

Our patient was a 34 year old male. Patients range from stillborn infants to a 95-year-old woman, with an average age of 24 years ${ }^{17}$.

In a series of 66 sporadic myxomas, the female-to-male ratio was 2.7:118 ${ }^{15}$ and $3: 1$ in one series ${ }^{19}{ }^{1}$ Cardiac myxomas have no typical presentation. Typically, patients are asymptomatic or present with nonspecific signs and symptoms. Some authors call heart neoplasms the great "masqueraders" ${ }^{20}$. The clinical features of LV myxoma are mostly caused by embolization and obstruction to LVOT and systemic manifestations as components of the classical triad, but rarely are present all. However, at least one of the triad symptoms is present. Arrhythmias, conduction disturbances, and LV dysfunction can also be seen $^{21,22}$.

Embolic phenomena in LV myxoma are more common than LA myxomas, occurring in $64 \%$ of patients with LV myxoma ${ }^{23}$.In our case ischemic stroke occurred as embolic phenomenon of myxoma and it was the first manifestation of otherwise asymptomatic myxoma.

Thrombus formation in LV is well-known complication in systolic heart failure (incidence 10-30\%) and after acute myocardial infarction (incidence 5-15\%) 24 .Hypercoagulable state can lead to ventricular thrombus formation even in the normal heart ${ }^{25}$. Main causes of Inherited thrombophilia are G1691A mutation of factor $\mathrm{V}$ gene, G20210A mutation of the prothrombin gene, antithrombin deficiency, protein $\mathrm{C}$ and protein $\mathrm{S}$ deficiency 26.LV thrombus formation is also associated with antiphospholipid antibody syndrome (APS)and hypereosinophilic syndrome ${ }^{27,28}$.Autoimmune disorders like Adamantiadis-Behcet's disease and Systemic lupus erythematosus(SLE), have been suggested to cause left ventricular thrombus formation 29,30 TEE of our patient demonstrated a thrombus attached to LV myxoma. The co-existence of SLE and Secondary APS could have been the contributing factors.

The most important method in myxoma preoperative diagnostics is transthoracic echocardiography. An additional diagnostic method in unclear diagnostic cases is transesophageal echocardiography 31,32 .

Echocardiography plays a key role in establishing the diagnosis of patients with cardiac myxomas and thrombi. The differentiation between myxomas and thrombi is important because of the distinct treatment strategy. But it is often difficult to triage one from the other.In our case the trans thoracic and TEE did not reveal the typical features of myxoma -a mobile mass attached by a stalk to the septum. But treatment with anticoagulation for a prolonged period only caused slight reduction in size of the mass; possibly due to resolution of its overlying thrombus.

In contrast to masses in ventricle masses in the atria were deemed to be thrombi because of associated spontaneous echo contrast, location in the left atrial appendage, mitral valvular disease or prosthesis, atrial fibrillation, congestive heart failure, and enlarged left atrial chamber. ${ }^{33}$

$\mathrm{CT}$ and MRI can be used to differentiate the kind of tumor in right parts of the heart. MRI allows determining more specific tumor localization, its size and spread, tumor position to adjacent organs, and also the histological structure as compared to $\mathrm{CT}$. ${ }^{34}$

A pedunculated thrombus moving throughout the cardiac cycle has a high tendency to embolize despite adequate anticoagulation ${ }^{35}$. Treatment for such thrombi has included thrombectomy, anticoagulation, or thrombolysis. Our patient was adequately anticoagulated and is now under the constant supervision of cardiac surgeon for further management.

Echocardiographies of our patient showed that the LV myxoma was sessile smooth surfaced and rounded. Cardiologist and cardiac surgeon both have opted for watchful observation. At present our patient is continuing medical management and is gradually improving neurologically and has not experienced any adverse events.

Most cases obtained complete recovery after tumor resection and histological confirmation can be obtained 36 . Our patient has not yet undergone surgical resection of the cardiac tumour. So histological confirmation of diagnosis has not been done. 


\section{Conclusion:}

Approximately $15 \%$ of all ischemic strokes (IS) occur in young adults and adolescents. The causes of Ischemic Stroke in the young are heterogeneous and can be relatively uncommon. We report a young adult suffering from ischemic stroke diagnosed with an isolated otherwise asymptomatic left ventricular myxoma with thrombus which is an extremely rare finding. Extensive search based on clinical clues also revealed SLE associated with Lupus nephritis and Secondary APS, Homocysteinemia, significant smoking history and history of recreational drug use; which are all contributors to ischemic stroke. But considering our patients clinical presentation and imaging findings LV myxoma seemed to have the major contribution in causation of stroke by embolization. The patient was managed conservatively, attempts were taken to treat modifiable vascular risk factors aggressively and healthy lifestyle especially abstinence from recreational drug use was advised.

\section{References:}

1. Grau AJ, Weimar C, Buggle F, et al. Risk factors, outcome, and treatment in subtypes of ischemic stroke: the German Stroke Data Bank. Stroke 2001;32:2559-66

2. Barinagarrementeria F, Figueroa T, Huebe J, Cantú C. Cerebral infarction in people under 40 years. Etiologic analysis of 300 cases prospectively evaluated. Cerebrovasc Dis 1996;6:75-79

3. Lipska K, Sylaja PN, Sarma PS, et al. Risk factors for acute ischaemic stroke in young adults in South India. J Neurol Neurosurg Psychiatry 2007;78:95963

4. Putaala J, Metso AJ, Metso TM, et al. Analysis of 1008 consecutive young patients aged 15 to 49 with first-ever ischemic stroke: the Helsinki Young Stroke Registry. Stroke 2009;40:1195-1203.

5. Bogousslavsky J, Pierre P. Ischemic stroke in patients under age 45. Neurol Clin 1992;10:11324

6. George MG, Tong X, Kuklina EV, Labarthe DR. Trends in stroke hospitalizations and associated risk factors among children and young adults, 19952008. Ann Neurol 2011;70:713-21.

7. Fields MC, Levine SR. Thrombophilias and stroke: diagnosis, treatment, and prognosis. J Thromb Thrombolysis $2005 ; 20: 113-26$
8. Brey RL. Antiphospholipid antibodies in young adults with stroke. J Thromb Thrombolysis 2005;20:105-112.

9. Morris JG, Singh S, Fisher M. Testing for inherited thrombophilias in arterial stroke. Can it cause more harm than good? Stroke 2010;41:2985-90

10. Sarjeant JM, Butany J, Cusimano RJ. Cancer of the heart: Epidemiology and management of primary tumors and metastases. Am J Cardiovasc Drugs 2003;3:407-21

11. Korkmaz AA, Tamtekin B, Onan B, Demir AS, Guden $M$, Uckurt $Y$. Combination of right atrial and left ventricular myxoma. Ann Thorac Surg 2010;89: e33-5.

12. Wilcox, B.R. and Carter, J.M. Left Ventricular Myxoma. Annals of Surgery, 1971;173:131-134.

13. Mazer, M. and Harrigan, P. Left Ventricular Myxoma, M-Mode and Two-Dimensional Echocardiographic Features. Am Heart J, 1987;104:875-77.

14. Keeling, I.M., Oberwalder, P., Anelli-Monti, M., Schuchlenz, H., Demel, U., Tilz, G.P., et al. Cardiac Myxomas:24 Years of Experience in 49 Patients. Eur J Cardiothorac Surg, 2002;22:971-7

15. Abad, C., Novoa, J., Deigado, A. and Alonso, A. Myxoma of the Left Ventricle. Tex Heart Ins J, 2014;41:395-400.

16. Prichard RW. Tumors of the heart: review of the subject and report of 150 cases. AMA Arch Pathol. 1951;51:98-128

17. Kitchin PL, Benson P. Left ventricular myxoma causing sudden death.Am J Forensic Med Pathol. 2015;36:58-60.

18. Zheng, J.J., Geng, X.G., Wang, H.C., Yan, Y. and Wang, H.Y. Clinical and Histopathological Analysis of 66Cases with Cardiac Myxoma. Asian Pac J Cancer, Prevention, 2013;14:1743-6.

19. R. Muthiah94[19] Yoon, D.H. and Roberts, W. Sex Distribution in Cardiac Myxomas. Am J Cardiol, 2002;90:563-565.

20. Rodriguez-Cruz, E. and Rao, P.S. Pediatric Cardiac Tumors, Clinical Presentation. Medscape 2016. Available at: e medicine. medscape.com accessed 20 Jan. 2017

21. Colucci W, Braunwald E. Primary tumors of the heart. In: Braunwald E, editor. Heart Disease: A 
Textbook of Cardiovascular Medicine. 4 th ed., Vol. 2. Philadelphia: WB Saunders; 1992. p. 1451

22. Buke A, Virmani R. Cardiac myxoma: A clinicopathologic study. Am J Clin Pathol 1993;100:671-80

23. Samdarshi TE, Mahan EF 3 rd, Nanda NC, Guthrie FW Jr, Bernstein IJ, Kirklin JW. Transesophageal echocardiographic diagnosis of multicentric left ventricular myxomas mimicking a left atrial tumor. J Thorac Cardiovasc Surg 1992;103:471-4.

24. Kalra A, Jang IK. Prevalence of early left ventricular thrombus after primary coronary intervention for acute myocardial infarction. J Thromb Thrombolysis 2000;10:133-6.

25. Verma AK, Alam M, Rosman HS, Brymer J, Keith F. Systemic embolization from thrombus in normal left ventricles. Chest 1988;93:441-2.

26. Lane DA, Mannucci PM, Bauer KA, Bertina RM, Bochkov NP, Boulyjenkov V, et al. Inherited thrombophilia: Part 1. Thromb Haemost 1996;76:651-62.

27. Aguilar JA, Summerson C. Intracardiac thrombus in antiphospholipid antibody syndrome. J Am Soc Echocardiogr 2000;13:873-5.

28. Ejima J, Ohmura I, Kaji Y, Tsuda Y, Kanaya S, Fujino T. Diffuse endocardial thrombus in left ventricle associated with a case of hypereosinophilic syndrome. Jpn Heart J 1991;32:267-72.

29. Vanhaleweyk G, el-Ramahi KM, Hazmi M, Sieck JO, Zaman L, Fawzy M. Right atrial, right ventricular and left ventricular thrombi in (incomplete) Behçet's disease. Eur Heart J 1990;11:957-9.

30. Barjatiya MK, Shah NK, Kothari SS, Shah PP, Trivedi HL. Spontaneous left ventricle cavity thrombus in a patient of systemic lupus erythematosus. J Assoc Physicians India 1992;40:195-6.

31. Keeling IM, Oberwalder $\mathrm{P}$, Anelli-Monti M, etal. Cardiac myxomas: 24 years of experience in 49 patients. Eur J Cardiothorac Surg 2002;22:971-7

32. Selkane C,Amahzoune B, Chavanis N, etal. Changing management of cardiac myxoma based on a series of 40 cases with long-term follow-up. Ann Thorac Surg 2003;76:1935-38.

33. Alam M,Sun I, Transesophageal echocardiographic evaluation of left atrial mass lesions. J Am Soc Echocardiogr. 1991 Jul-Aug;4(4):323-30.

34. Hoey ET, Shahid M, Ganeshan A, Baijal S, Simpson $\mathrm{H}$, Watkin RW et al. MRI assessment of cardiac tumours: part 1, multiparametric imaging protocols and spectrum of appearances of histologically benign lesions.Quant Imaging Med Surg. 2014 Dec; 4(6): 478-88.

35. Glikson M, Agranat O, Ziskind Z, Kaplinski E, Vered Z. From swirling to a mobile, pedunculated mass The evolution of left ventricular thrombus despite full anticoagulation. Echocardiographic demonstration. Chest 1993;103:281-3

36. Kong Y, Li H, MM, Wang J, Chai Y, Hou W, Zhang N. Left Ventricular Myxoma Leading to Stroke-A Rare Case Report. Medicine (Baltimore). 2015 Nov; 94(45): e1913. 